\title{
Tumor Preserving Medical Image Compression
}

\author{
Priyanka Somvanshi \\ Vishwakarma Institute Of \\ Technology \\ City- Pune, Maharashtra \\ Country- India, Pin- 411037
}

\author{
Usham Dias \\ Vishwakarma Institute of \\ Technology \\ City- Pune, Maharashtra \\ Country- India, Pin- 411037
}

\author{
Rupali Tornekar \\ Vishwakarma Institute Of \\ Technology \\ City- Pune, Maharashtra \\ Country- India, Pin- 411037
}

\begin{abstract}
Medical imaging involves handling of huge volumes of DICOM images. Main agenda is to compress the images without compromising on the quality of the image. In this paper, a comparative analysis of different compression techniques is made for medical DICOM images. Lossless compression based on General indexing and Huffman gave maximum compression ratio 1.6 and 1.85 . The proposed lossy compression is based on $\mathrm{db} 1, \mathrm{db} 2$ wavelet at single level decomposition. The proposed technique is computationally efficient since it uses a simple algorithm, at the same time achieving good PSNR, compression ratio and bits per pixel (bpp). The PSNR achieved with the proposed algorithm is always above $54.5 \mathrm{db}$ across all test images. The results obtained clearly indicate that the proposed technique preserves the tumor region, thus not affecting medical diagnosis. Thus further processing like segmentation, tumor detection and classification can be applied on these compressed images.
\end{abstract}

\section{Keywords}

Tumor detection, lossy compression, Wavelet, Huffman

\section{INTRODUCTION}

MRI (Magnetic resonance imaging) is the most widely used method in medical diagnosis. In the medical imaging MRI images are voluminous. Each image requires a large amount of storage and collectively a patient's record may require many images to be stored. So to reduce the storage space compression is done. In case of medical imaging the most important requirement for any compression technique is to preserve the ROI (region of interest) which in our case is the tumor part so that medical diagnosis must not be affected thus maintaining the quality of the image. An image compression technique can be classified as lossless or lossy.

In lossless compression no data is lost i.e. image is reconstructed perfectly. As compared to the lossless compression technique the lossy techniques are more efficient in terms of compression ratio. In this the required image characteristics are usually preserved in the coefficients of domain space in which original image is transformed into. In DWT (Discrete Wavelet Transform) image compression the wavelet coefficients i.e. approximation coefficients keep all the information needed for reconstructing the medical image. To achieve maximum compression ration only the approximation coefficients are saved discarding others. The different parameters to judge the performance of the lossy compression technique is PSNR, compression ratio, bits per pixel, etc.

In the next section, the related work is briefed. In Section 3, the algorithms for lossless and lossy compression are described.
Section 4 contains experimental results. We offer our conclusions in Section 5.

\section{RELATED WORK}

Ruchika et al. [1] proposed a method in which the redundancy of the medical image and DWT coefficients are reduced through thresholding and further through Huffman encoding.

In [2], Two Component Medical Image Compression Technique is implemented where some of the slices in a sequence are represented by JPEG data and some of the slices are represented by SPIHT data.

Paper [3] gives analysis of efficient wavelet based volumetric image compression.

In this paper we propose a method giving better results in terms of the compression ratio (CR), PSNR and bits per pixel (bpp).

\section{IMPLEMENTATION}

\subsection{Lossless technique}

In General indexing technique, the histogram of the image is computed and the number of non-zero intensity values is found. The number of bits to encode is $\log 2(\mathrm{~N})$, where $\mathrm{N}$ is the number of non-zero intensity, which is fixed across all pixels.

In Huffman coding, the histogram of the image is found and probability of intensity values is calculated. Based on these probabilities the standard Huffman algorithm is applied to generate the variable length code.

\subsection{Lossy Technique}

In image compression, transform coding techniques the most popular method used is discrete wavelet transform (DWT). Wavelet transform provides the time-frequency analysis simultaneously as it provides multi-resolution analysis.

The discrete wavelet transform is implemented using multirate filter banks. These filters divide a signal frequency into subbands. At each level of decomposition the approximation coefficients are generated from low pass filter and the detail coefficients from high pass filter. DWT analyzes an image across rows and columns so as to separate the horizontal, vertical, diagonal details as shown in Fig.1.

In case of single level decomposition at first stage the rows are filtered using low and high pass filters. The filtering is done using the 1-D convolution with filter coefficients; this is followed by the downsampling with factor 2 . In second stage filtering is done on columns followed by downsampling with factor 2 giving the four subbands LL, HL, LH, HH. The Fig.1 shows the single level decomposition. 

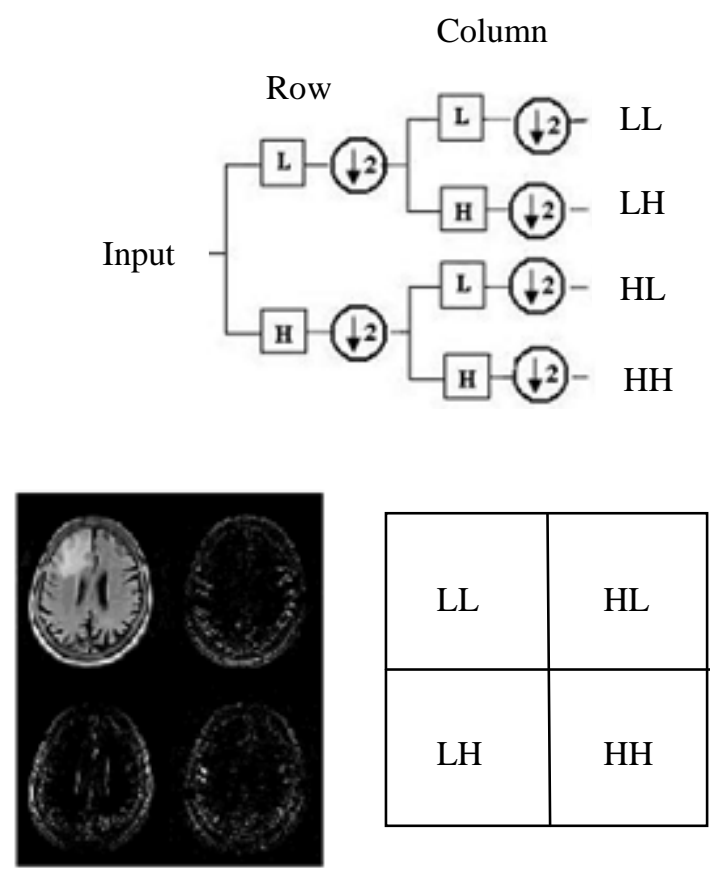

Fig.1 Single level decomposition

The single level decomposition shown in Fig.1 clearly indicates that the approximation coefficient (LL) preserves most of the information including the tumor region hence we can discard other detail information like horizontal, vertical and diagonal to achieve good compression ratio. In this paper two wavelets are examined: Daubechies 1 i.e. Haar and Daubechies 2. The wavelet functions for the Haar and Daubechies 2 as shown in Fig.2.

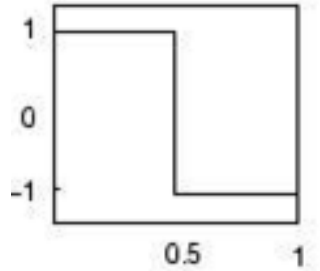

Haar

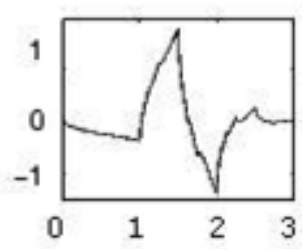

Daubechies 2
Fig.2 Wavelet functions for the Haar and Daubechies 2

The proposed wavelet based image compression algorithm is explained below:

1. Read the DICOM image

2. Decompose the image using the DWT

3. Scale the approximate coefficients

i. If the maximum coefficient value is above 1000 then scale by factor 100 .

ii. If the maximum coefficient value is below 1000 then scale by factor 10 .

4. These scaled coefficients are then quantized by thresholding.

5. The approximate coefficients are then encoded using Huffman coding technique.
The inverse discrete wavelet transform (IDWT) reconstructs the scaled and thresholded approximate coefficient. The reconstructed image based on the proposed method is shown in Fig.3, which shows that the tumor part is preserved after the reconstruction. For viewing purpose only image 1, 2, 11 and 12 are shown.
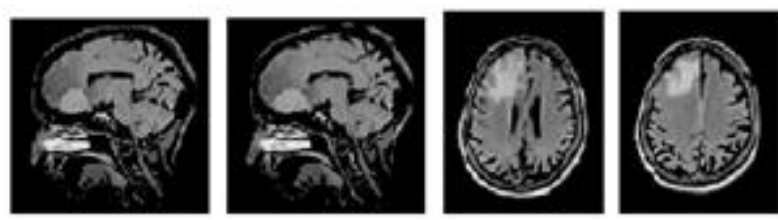

Reconstructed image using db1
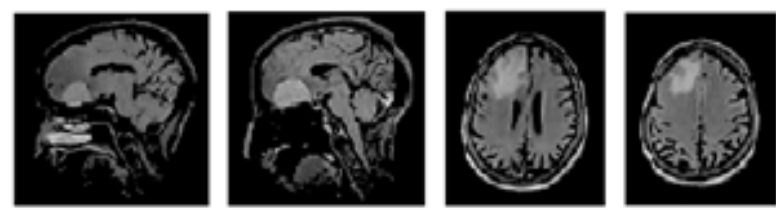

Reconstructed image using $\mathrm{db} 2$

Fig.3 Reconstructed image based on the proposed method

\section{EXPERIMENTAL RESULTS}

The images used to test the performance of the Medical Image compression techniques are shown in Fig.4. These images are obtained from Gundiyal radio diagnostic centre, Amravati, Maharashtra, India. All the images used for testing are 16-bit unsigned integer, DICOM images. The results for lossless compression based on General Indexing technique and Huffman coding are shown in Table 1 and 2 respectively. The results for lossy compression are based on $\mathrm{db} 1, \mathrm{db} 2$ wavelets is shown in table 3,4 respectively. The codes were executed on an Intel Core i5-2430 processor with 4GB RAM memory.

The parameters used to evaluate the results are defined as below:

\section{Compression Ratio (CR):}

$\mathbf{C R}=\frac{\mathrm{n} 1}{\mathrm{n} 2}$

where $\mathrm{n} 1$ is the number of bits to represent the original image and $\mathrm{n} 2$ is the number of bits to represent the encoded image.

2. Peak Signal to Noise Ratio (PSNR):

PSNR $=10 \log _{10} \frac{\left(2^{\mathrm{r}}-1\right)^{2}}{\mathrm{MSE}}$

where $r$ is the number of bits required to represent the original image. In our case $r=16$.

3. Bits Per Pixel (bpp):

$\mathbf{B p p}=\frac{\mathrm{b} 1}{\mathrm{~b} 2}$

where $b 1$ is the number of bits to represent the encoded image and b2 is the total number of pixels in the original image. 
The maximum compression ratio achieved using simple indexing is 1.6. The average encoding and decoding time across all the 12 test images is 478.4738 and $69.6645 \mathrm{sec}$ respectively. The maximum compression ratio achieved using Huffman encoding is 1.8568. The average encoding and decoding time across all the 12 test images is 680.9699 and $1001.926 \mathrm{sec}$ respectively. Since both the techniques are lossless they perfectly reconstruct the image.

The maximum compression ratio achieved using db1 based wavelet compression is 40.7784 . The average encoding time, decoding time, bits per pixel and PSNR across all the 12 test images is $6.6321 \mathrm{sec}, 1.5553 \mathrm{sec}, 0.55592 \mathrm{bpp}$ and 56.78475 $\mathrm{db}$ respectively.

The maximum compression ratio achieved using $\mathrm{db} 2$ based wavelet compression is 40.1415 . The average encoding time, decoding time, bits per pixel and PSNR across all the 12 test images is $6.6607 \mathrm{sec}, 1.5559 \mathrm{sec}, 0.54659 \mathrm{bpp}$ and 56.91690 $\mathrm{db}$ respectively.

Fig. 3 shows the reconstructed images based on $\mathrm{db} 1, \mathrm{db} 2$ wavelet compression. The reconstructed images clearly preserve the quality of the image and more importantly the tumor region.

\section{CONCLUSION}

The quantitative analysis of the compression techniques clearly shows the ineffectiveness of the lossless techniques to achieve high compression ratios and less time for computation. The proposed technique based on single level decomposition uses scaling and thresholding of approximation coefficients. Thus the time for encoding and decoding required is less. The PSNR achieved is above $54.5 \mathrm{db}$ across all the test images, for wavelets namely $\mathrm{db} 1, \mathrm{db} 2$. The maximum PSNR, CR and minimum bpp achieved by [1] is comparatively lower than that achieved by our proposed method using $\mathrm{db} 1$ and $\mathrm{db} 2$. The proposed algorithm also provides superior performance in terms of compression rate and PSNR compared to [2]. The formula used by [3] to calculate CR is different from the one used in this paper; using the formula given in [3] maximum CR we achieved is 97.54 which is much higher than the maximum achieved i.e. 79.6038 in paper [3] for MRI images. The results obtained by our proposed method clearly demonstrate that the superior quality images can be obtained preserving the tumor region. Hence further processing like segmentation, tumor detection and classification can be applied on these compressed images.

\section{REFERENCES}

[1] Ruchika, Mooninder Singh, Anant Raj Singh, "Compression of Medical Images Using Wavelet Transforms", International Journal of Soft Computing and Engineering (IJSCE) ISSN: 2231-2307, Volume-2, Issue-2, May 2012

[2] K. Vidhya, and Dr. Shenbagadevi, "A Two Component Medical Image Compression Technique", International
Journal of Recent Trends in Engineering, Vol. 1, No. 1, May 2009

[3] Krishna Kumar, Basant Kumar, Rachna Shah, "Analysis of Efficient Wavelet Based Volumetric Image Compression", International Journal of Image Processing (IJIP), Volume (6), Issue (2), 2012.

[4] R.Sumalatha, M.V.Subramanyam, "Medical Image Compression Using Multiwavelets for Telemedicine Applications", International Journal of Scientific \& Engineering Research Volume 2, ISSN 2229-5518, Issue 9, September-2011

[5] Sonja Grgic, Mislav Grgic, Branka Zovko-Cihlar, "Performance Analysis of Image Compression Using Wavelets", IEEE transactions on industrial electronics, vol. 48, no. 3, June 2001

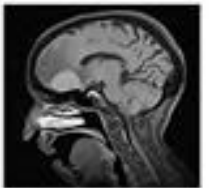

Image1

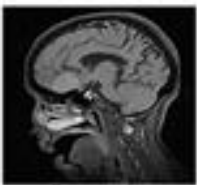

Image4

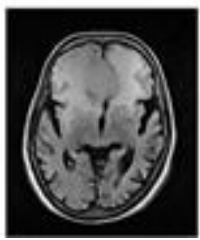

Image7

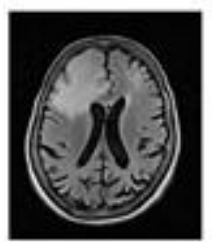

Image10

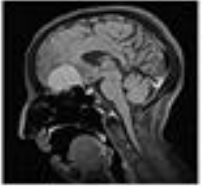

Image2

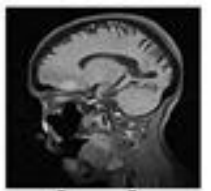

Image5

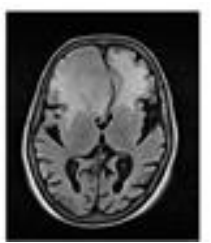

Image8

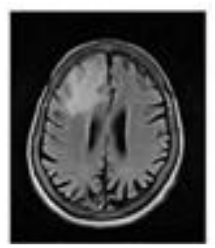

Image11

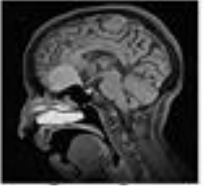

Image3

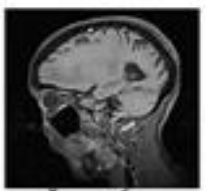

Image6

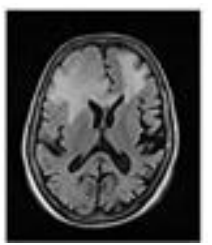

Image9

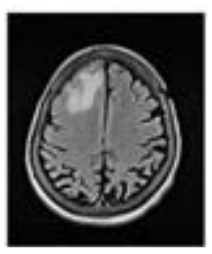

Image12
Fig.4 Medical DICOM MRI Image dataset 


\section{RESULT TABLE}

Table 1: Results for General Indexing Technique for compression

\begin{tabular}{|c|c|c|c|c|c|}
\hline $\begin{array}{c}\text { Uncompressed } \\
\text { Size (bits) }\end{array}$ & $\begin{array}{c}\text { Compressed } \\
\text { Size (bits) }\end{array}$ & $\begin{array}{c}\text { Reduction } \\
\text { (Bits) }\end{array}$ & $\begin{array}{c}\text { Compression } \\
\text { Ratio }\end{array}$ & $\begin{array}{c}\text { Encoding } \\
\text { Time (sec) }\end{array}$ & $\begin{array}{c}\text { Decoding } \\
\text { Time(sec) }\end{array}$ \\
\hline 1048576 & 655360 & 393216 & 1.6 & 562.0862 & 77.6191 \\
\hline 1048576 & 655360 & 393216 & 1.6 & 562.2000 & 77.6724 \\
\hline 1048576 & 720896 & 327680 & 1.4545 & 616.4801 & 77.8448 \\
\hline 1048576 & 655360 & 393216 & 1.6 & 563.5485 & 78.1681 \\
\hline 1048576 & 655360 & 393216 & 1.6 & 566.2837 & 76.2985 \\
\hline 1048576 & 655360 & 393216 & 1.6 & 560.8659 & 76.4253 \\
\hline 819200 & 563200 & 256000 & 1.4545 & 372.4082 & 60.4516 \\
\hline 819200 & 563200 & 256000 & 1.4545 & 376.7383 & 59.9724 \\
\hline 819200 & 563200 & 256000 & 1.4545 & 372.3132 & 60.0037 \\
\hline 819200 & 563200 & 256000 & 1.4545 & 373.6025 & 59.5371 \\
\hline 819200 & 563200 & 256000 & 1.4545 & 403.2066 & 64.8760 \\
\hline 819200 & 563200 & 256000 & 1.4545 & 411.9523 & 67.1044 \\
\hline
\end{tabular}

Table 2: Results using Huffman Coding

\begin{tabular}{|c|c|c|c|c|c|}
\hline $\begin{array}{c}\text { Uncompressed } \\
\text { Size (bits) }\end{array}$ & $\begin{array}{c}\text { Compressed } \\
\text { Size (bits) }\end{array}$ & $\begin{array}{c}\text { Reduction } \\
\text { (Bits) }\end{array}$ & $\begin{array}{c}\text { Compression } \\
\text { Ratio }\end{array}$ & $\begin{array}{c}\text { Encoding } \\
\text { Time (sec) }\end{array}$ & $\begin{array}{c}\text { Decoding } \\
\text { Time(sec) }\end{array}$ \\
\hline 1048576 & 577017 & 471559 & 1.8172 & 768.5243 & 1050.0715 \\
\hline 1048576 & 565831 & 482745 & 1.8531 & 713.7882 & 850.46751 \\
\hline 1048576 & 581776 & 466800 & 1.8024 & 776.9908 & 1072.0843 \\
\hline 1048576 & 576830 & 471746 & 1.8178 & 792.5192 & 975.83228 \\
\hline 1048576 & 570095 & 478481 & 1.8393 & 751.3045 & 844.28525 \\
\hline 1048576 & 564710 & 483866 & 1.8568 & 745.5834 & 853.56505 \\
\hline 819200 & 466408 & 352792 & 1.8531 & 619.8670 & 1147.9347 \\
\hline 819200 & 468265 & 350935 & 1.7494 & 615.1288 & 1073.6692 \\
\hline 819200 & 456515 & 362685 & 1.7944 & 606.1636 & 1108.7721 \\
\hline 819200 & 451380 & 367820 & 1.8148 & 601.3731 & 1064.2832 \\
\hline 819200 & 459898 & 359302 & 1.7812 & 601.5242 & 997.59101 \\
\hline 819200 & 453047 & 366153 & 1.8082 & 578.8711 & 984.55619 \\
\hline
\end{tabular}


Table 3: Results using 'db1' wavelet for compression

\begin{tabular}{|c|c|c|c|c|c|c|c|}
\hline $\begin{array}{c}\text { Uncompressed } \\
\text { Size (bits) }\end{array}$ & $\begin{array}{c}\text { Compressed } \\
\text { Size (bits) }\end{array}$ & $\begin{array}{c}\text { Reduction } \\
\text { (Bits) }\end{array}$ & $\begin{array}{c}\text { Compression } \\
\text { Ratio }\end{array}$ & $\begin{array}{c}\text { Encoding } \\
\text { Time (sec) }\end{array}$ & $\begin{array}{c}\text { Decoding } \\
\text { Time(sec) }\end{array}$ & $\begin{array}{c}\text { Bits per pixel } \\
\text { (BPP) }\end{array}$ & PSNR (db) \\
\hline 1048576 & 33993 & 1014583 & 30.84682 & 7.848655 & 1.513111154 & 0.518692017 & 56.2589 \\
\hline 1048576 & 33207 & 1015369 & 31.57696 & 7.544755 & 1.46611489 & 0.506698608 & 57.34741 \\
\hline 1048576 & 34130 & 1014446 & 30.723 & 7.685736 & 1.513738906 & 0.520782471 & 55.72597 \\
\hline 1048576 & 33470 & 1015106 & 31.32883 & 7.771816 & 1.555558359 & 0.51071167 & 56.02951 \\
\hline 1048576 & 28299 & 1020277 & 37.05346 & 7.057945 & 1.359156812 & 0.431808472 & 55.00055 \\
\hline $\mathbf{1 0 4 8 5 7 6}$ & $\mathbf{2 5 7 1 4}$ & $\mathbf{1 0 2 2 8 6 2}$ & $\mathbf{4 0 . 7 7 8 4 1}$ & $\mathbf{6 . 8 1 7 8 7 9}$ & $\mathbf{1 . 3 0 3 1 0 7 3 2 7}$ & $\mathbf{0 . 3 9 2 3 6 4 5 0 2}$ & $\mathbf{5 4 . 5 1 2 4 1}$ \\
\hline 819200 & 33571 & 785629 & 24.40201 & 5.974884 & 1.753992401 & 0.655683594 & 57.73823 \\
\hline 819200 & 33136 & 786064 & 24.72236 & 5.924388 & 1.738727093 & 0.6471875 & 57.43998 \\
\hline 819200 & 33009 & 786191 & 24.81747 & 5.957963 & 1.69516208 & 0.644707031 & 57.73033 \\
\hline 819200 & 32415 & 786785 & 25.27225 & 5.843904 & 1.668441162 & 0.633105469 & 57.95229 \\
\hline 819200 & 31789 & 787411 & 25.76992 & 5.725232 & 1.574762081 & 0.620878906 & 57.75982 \\
\hline 819200 & 30128 & 789072 & 27.19065 & 5.431493 & 1.522110494 & 0.5884375 & 57.92162 \\
\hline
\end{tabular}

Table 4: Results using 'db2' wavelet for compression

\begin{tabular}{|c|c|c|c|c|c|c|c|}
\hline $\begin{array}{c}\text { Uncompressed } \\
\text { Size (bits) }\end{array}$ & $\begin{array}{c}\text { Compressed } \\
\text { Size (bits) }\end{array}$ & $\begin{array}{c}\text { Reduction } \\
\text { (Bits) }\end{array}$ & $\begin{array}{c}\text { Compression } \\
\text { Ratio }\end{array}$ & $\begin{array}{c}\text { Encoding } \\
\text { Time (sec) }\end{array}$ & $\begin{array}{c}\text { Decoding } \\
\text { Time(sec) }\end{array}$ & $\begin{array}{c}\text { Bits per pixel } \\
\text { (BPP) }\end{array}$ & PSNR (db) \\
\hline 1048576 & 31109 & 1017467 & 33.70652 & 7.311287 & 1.298308107 & 0.474685669 & 55.42735 \\
\hline 1048576 & 33588 & 1014988 & 31.21877 & 7.747085 & 1.445256692 & 0.512512207 & 57.864 \\
\hline 1048576 & 34512 & 1014064 & 30.38294 & 7.961583 & 1.622792767 & 0.526611328 & 56.10582 \\
\hline 1048576 & 33825 & 1014751 & 31.00003 & 7.865699 & 1.647248135 & 0.51612854 & 56.43271 \\
\hline 1048576 & 28689 & 1019887 & 36.54976 & 7.209151 & 1.393387656 & 0.437759399 & 55.19587 \\
\hline $\mathbf{1 0 4 8 5 7 6}$ & $\mathbf{2 6 1 2 2}$ & $\mathbf{1 0 2 2 4 5 4}$ & $\mathbf{4 0 . 1 4 1 4 9}$ & $\mathbf{6 . 9 2 9 1 6 4}$ & $\mathbf{1 . 3 4 1 7 4 5 2 6}$ & $\mathbf{0 . 3 9 8 5 9 0 0 8 8}$ & $\mathbf{5 4 . 6 0 6 2 4}$ \\
\hline 819200 & 32513 & 786687 & 25.19608 & 5.865098 & 1.826886408 & 0.635019531 & 57.79099 \\
\hline 819200 & 32304 & 786896 & 25.35909 & 5.984411 & 1.688300431 & 0.6309375 & 57.65296 \\
\hline 819200 & 31965 & 787235 & 25.62803 & 5.898553 & 1.672112739 & 0.624316406 & 57.78141 \\
\hline 819200 & 31245 & 787955 & 26.21859 & 5.786592 & 1.615618953 & 0.610253906 & 57.8949 \\
\hline 819200 & 30600 & 788600 & 26.77124 & 5.709383 & 1.591121678 & 0.59765625 & 57.59105 \\
\hline 819200 & 30450 & 788750 & 26.90312 & 5.660963 & 1.528293076 & 0.594726563 & 58.65953 \\
\hline
\end{tabular}

